\title{
Pressure-controlled ventilation-volume guaranteed (PCV-VG) for postoperative pulmonary complications: a protocol for a systematic review with meta-analysis and trial sequential analysis
}

Jianqiao Zheng

Sichuan University West China Hospital https://orcid.org/0000-0002-8091-1837

Li Du

Sichuan Cancer Hospital and Research Institute: Sichuan Cancer Hospital and Institute

Xiaoqian Deng

Sichuan University West China Hospital

Lu Zhang

Sichuan University West China Hospital

Jia Wang

Sichuan University West China Hospital

Guo Chen (D Anesthesiology_SCU@163.com )

Sichuan University West China Hospital

\section{Protocol}

Keywords: pressure-controlled ventilation-volume guaranteed, intraoperative, mechanical ventilation, meta-analysis, randomized controlled trial

Posted Date: April 20th, 2021

DOI: https://doi.org/10.21203/rs.3.rs-435510/v1

License: (a) (i) This work is licensed under a Creative Commons Attribution 4.0 International License. Read Full License 


\section{Abstract}

\section{Background}

More than 300 million major surgical procedures are performed worldwide yearly. Above $30 \%$ of patients undergoing surgery with mechanical ventilation may experience postoperative pulmonary complications (PPCs). PPCs are the main cause of perioperative morbidity and mortality and it can be decreased by optimizing the mechanical ventilation. Pressure-controlled ventilation-volume guaranteed (PCV-VG) is a new ventilation mode, which combines the advantages of volume-controlled ventilation and pressurecontrolled ventilation, might reduce PPCs. The efficacy of PCV-VG for PPCs has not yet been systematically reviewed. Hence, we will conduct a systematic review to evaluate the efficacy of PCV-VG for PPCs. The aim of this protocol is to investigate the benefits of PCV-VG versus conventional ventilation mode for PPCs.

\section{Methods}

We will search PubMed, Web of Science, Cochrane Library, Ovid medline, Embase, China National Knowledge Infrastructure, Chinese BioMedical Literature, Wanfang and VIP databases from their inception until May 2021, to identify randomised controlled studies using related keywords. Simultaneously, clinical registration tests and gray literature will also be retrieved. Studies published in English or Chinese will be considered. The primary outcome will be the incidence of PPCs, secondary outcomes will be intraoperative parameters of respiratory and hemodynamic function. Data synthesis/statistical analyses will be performed using the Review Manager software (version 5.4) and Stata (version 16). Heterogeneity will be assessed by the standard chi-square test and $\mathrm{I}^{2}$ statistic. Two authors will independently search, extract data from and assess the risk bias of included studies according the Cochrane risk of bias tool. Trial sequential analysis will be used to control the risks of random errors. Funnel plots and Egger's regression test will be used to assess the publication bias. Certainty of the evidence will be assessed by modified Jadad Scale.

\section{Discussion}

This study will systematically and comprehensively search literature and integrate evidence on the efficacy of PCV-VG for PPCs. Our results will help clinical decision-making and support the development of clinical practice guidelines.

\section{Background}

More than 300 million major surgical procedures are performed worldwide each year [1]. In patients accepting surgery lasting over 2 hours under general anesthesia and mechanical ventilation, over $30 \%$ patients may experience postoperative pulmonary complications (PPCs) [2]. PPCs have a strong negative impact on postoperative recovery through increased morbidity, prolonged hospital stays, and early mortality $[3,4]$. The incidence of PPCs in the surgical population ranges from $11-33 \%[2,5]$. The 30 -day 
mortality rate after major surgery of patients developing PPCs was $14-30 \%$, higher than the rate (ranges from $0.2-3 \%$ ) of those without a PPCs [6]. Considering more than 300 million surgical procedures are performed worldwide per year, decreasing the incidence of PPCs could reduce the global morbidity and mortality, improve the quality of medical care and decrease the health system costs $[1,7]$.

Mechanical ventilation during general anesthesia has the potential risk to cause ventilator-associated lung injury (VALI) and therefore, lead to PPCs [8]. According to an international expert panel-based consensus, prevention of PPCs is an important therapeutic and economic goal, which may in part be accomplished by the optimization of mechanical ventilation $[9,10]$.

Volume-controlled ventilation (VCV) and pressure-controlled ventilation (PCV) are the most common mechanical ventilation mode during anesthesia. In VCV mode, ventilator delivers a target volume with a constant flow, the preset tidal volume and minute ventilation can be guaranteed during ventilation. However, this flow pattern may lead to higher peak inspiratory pressure (PIP) in response to reduced compliance or increased resistance. High inspiratory pressure can lead to shear stress injury, barotrauma and volutrauma to the alveoli, increasing the incidence of micro atelectasis and causing imbalanced distribution of pulmonary gases and inflammatory mediator release, which are the characteristic of ventilator-associated lung injury [11-14].

Compared with VCV, PCV provides tidal volume at a preset pressure with decelerating flow, and the tidal volume can be changed depending on lung compliance. ${ }^{15}$ This flow pattern can produce lower airway pressure, maintain the uniform distribution of pulmonary gas, improve oxygenation and reduce the incidence of barotrauma $[16,17]$. However, PCV has the limitation of variable tidal volume and minute ventilation according to the respiratory system compliance and resistance. So, there is always a risk of hypoventilation or hyperventilation, especially during transition from the supine to the trendelenburg position, or during pneumoperitoneum with carbon dioxide $\left(\mathrm{CO}_{2}\right)[18,19]$.

Pressure-controlled volume guaranteed (PCV-VG) ventilation, also called dual-controlled ventilation or pressure regulated volume controlled (PRVC) ventilation is a new mode introduced in the field of anesthesiology recently. In this mode, ventilator parameters are automatically changed with patient's each breath to offer the preset tidal volume without increasing airway pressures [20,21]. So, it combines the advantages of VCV and PCV [22]. PCV-VG provides a lower incidence of barotraumas especially in neonates, elderly patients, obese patients and patients with acute lung injury or chronic obstructive pulmonary diseases [23-26]. Compared to the conventional modes, PCV-VG might improve arterial oxygenation, reduce inflammatory factors, decrease acute lung injury (ALI) for thoracic surgery and laparoscopic surgery [27-33]. However, whether intraoperative PCV-VG could reduce PPCs remains unclear [34-36].

Therefore, we present this systematic review protocol designed to quantitatively investigate the efficacy of PCV-VG for intraoperative mechanical ventilation, especially to investigate the influence of PCV-VG on PPCs. 


\section{Methods/design}

\section{Study registration}

This systematic review has been registered with PROSPERO (registration number: CRD 42020207555). This protocol was prepared according to the Preferred Reporting Items for Systematic Reviews and MetaAnalyses Protocols (PRISMA-P) guidelines. The review will be conducted in accordance with the PRISMA guidelines [37,38]. The study is expected to begin searching in May 2021 and end in November 2021.

\section{Eligibility criteria}

\section{Inclusion criteria}

Type of studies We will include randomized controlled trials (RCTs) that evaluated the PCV-VG, dualcontrolled ventilation or PRVC ventilation for intraoperative mechanical ventilation in adults (aged 18 years old or over). Only studies published in English or Chinese will be included. No time limits will be applied to the searches.

Types of participants Adult Participants (18 years of age or older) undergoing operations requiring intraoperative mechanical ventilation will be included. Intraoperative mechanical ventilation with PCV-VG, PRVC or dual-controlled ventilation will be included. There will be no limits on study participants in terms of gender, ethnicity and types of surgery.

Types of interventions In the intervention group, participants had to have received intraoperative mechanical ventilation with PCV-VG, PRVC or dual-controlled ventilation.

Types of comparators The comparator group will be the participants received intraoperative mechanical ventilation with conventional mechanical ventilation modes (Such as VCV or PCV)

\section{Type of outcome measurements}

Primary outcomes The primary outcome will be the incidence of PPCs, expressed as risk ratio (RR). PPCs being defined as the composite of any of respiratory infection, respiratory failure, pleural effusion, atelectasis, or pneumothorax, as defined in the definition of postoperative respiratory complications from European Perioperative Clinical Outcome (EPCO) consensus statement and the recent consensus definitions $[39,40]$.

Secondary outcome The secondary outcomes will be:

1. Intraoperative gas exchange parameters: arterial partial pressure of oxygen $\left(\mathrm{PaO}_{2}\right)$; arterial oxygen saturation $\left(\mathrm{SaO}_{2}\right)$; arterial partial pressure of carbon dioxide $\left(\mathrm{PaCO}_{2}\right)$; pressure of end-tidal carbon dioxide $\left(\mathrm{PEtCO}_{2}\right)$; oxygenation index $\left[\mathrm{PaO}_{2}\right.$ / fraction of inspiration $\left.\mathrm{O}_{2}\left(\mathrm{FiO}_{2}\right)\right]$; Physiologic Deadspace to Tidal Volume [Dead space volume $\left(\mathrm{V}_{\mathrm{D}}\right) /$ Tidal volume $\left(\mathrm{V}_{\mathrm{T}}\right)$ ]. 
2. Intraoperative respiratory parameters: peak inspiratory pressure $\left(P_{\text {peak }}\right)$, mean inspiratory pressure $\left(P_{\text {mean }}\right)$, plateau inspiratory pressure $\left(P_{\text {plateau }}\right)$, pulmonary dynamic compliance $\left(C_{d y n}\right)$.

3. Intraoperative hemodynamic parameters: mean arterial pressure (MAP), heart rate (HR), central venous pressure (CVP).

\section{Exclusion criteria}

Studies with the following situations will be excluded: (a) participants undergoing emergency surgery; (b) participants with respiratory complications before surgery; (c) studies with insufficient data or without an effective classification; duplicated data or data that cannot be extracted; (d) crossover trials, cluster-RCTs, quasi-RCTs, uncontrolled trial, animal study, review, comment, case report, case series, non-clinical study.

\section{Databases and search strategy}

The following electronic databases will be searched from inception: English database including-PubMed, Web of Science, Cochrane Library, Ovid MEDLINE and Embase; Chinese database including-China National Knowledge Infrastructure (CNKI), Chinese BioMedical Literature, Wanfang database and VIP Database. These electronic databases will be searched from inception to May 2021. In addition, we will scrutinize the list of references, relevant conference literature, dissertations and trial registry database (WHO International Clinical Trials Registry Platform and Clinical Trials.gov) to overcome the publication bias due to identify additional studies.

The search strategy for PubMed (as an example) is shown in table 1. The following search terms will be used singly or as combinations (MeSH terms and free words): pressure-controlled volume guaranteed, PCV-VG, dual controlled ventilation, pressure regulated volume controlled, PRVC, intraoperative and randomized controlled trial. The search terms will be translated into Chinese for study identification in Chinese databases. Prior to final publication, we will perform a new search in the databases to check if any studies were published during the elaboration of the systematic review. The preliminary search strategy of other electronic databases is given in (online supplementary additional file 1).

Table 1 Search strategy applied in PubMed 
\#1 $\quad((((((()((((($ Pressure controlled volume guaranteed[Title/Abstract]) OR (Pressure-controlled volume-guaranteed[Title/Abstract])) OR (Pressure-controlled Ventilation with Volume Guarantee[Title/Abstract])) OR (Pressure-controlled volume guaranteed[Title/Abstract])) OR (pressurecontrolled ventilation-volume guaranteed[Title/Abstract])) OR (pressure-controlled ventilation volume guaranteed[Title/Abstract])) OR (Pressure-controlled ventilation with volumeguarantee[Title/Abstract])) OR (pressure support ventilation with volume guarantee[Title/Abstract])) OR (pressure support ventilation plus volume guarantee[Title/Abstract])) OR (pressure support plus volume guarantee[Title/Abstract])) OR (Volume-guaranteed pressure-support[Title/Abstract])) OR (volume-guaranteed pressure-regulated[Title/Abstract])) OR (PCV-VG[Title/Abstract])) OR (Dual controlled ventilation[Title/Abstract])) OR (Pressure Regulated Volume Controlled[Title/Abstract])) OR (PRVC[Title/Abstract])

\#2 (Surgical Procedures, Operative [MeSH Major Topic]) OR ((((((Operat*) OR (surger*)) OR (surgical)) OR (intraoperat*)) OR (intra-operat*)) OR (Peri-operat*)) OR (Perioperat*))

\#3 ((((((controlled clinical trial [Publication Type]) OR (randomized controlled trial [Publication Type])) OR (randomized [Title/Abstract])) OR (randomized [Title/Abstract])) OR (Placebo [Title/Abstract])) OR (randomly [Title/Abstract])) OR (Clinical trial [Title])

\#4 (animals [MeSH Terms]) NOT ((human [MeSH Terms]) AND (animals [MeSH Terms]))

\#5 \#1 and \#2 and \#3 not \#4

*Represents multiple characters

\section{Study selection}

Two authors (JQZ and LD) will independently perform study selection based on pre-designed eligibility criteria. All searched records will be imported into citation management system (Endnote X9), and we will filter and remove all duplicates. First, all studies will be identified by screening titles/abstracts, and irrelevant records will be eliminated. Then, the full text of potential studies will be obtained and checked against all pre-designed inclusion criteria. If divergences occur, a third author will help determine and solve them to reach a final decision about whether the trial meets the eligibility criteria through discussion or consensus meeting.

Two reviewers (JQZ and LD) will be required to screen the retrieved studies independently. We will import the searched records into citation management system (Endnote X9), filter and remove all duplicates. Briefly, they will exclude studies not matching the inclusion criteria by reading title and abstracts. Then they will read the full text of each study to select those meeting the inclusion criteria. Any disagreements will be resolved through discussion with a third reviewer (JW). A fourth reviewer (LZ) will check all procedures before approving the data extraction. The entire study selection process is shown in the flow diagram (figure 1).

\section{Data extraction and management}

Two reviewers (JQZ and XQD) will extract data from the included studies independently following a previously designed standard data acquisition excel form. The form will include the basic information (e.g., first author, title, journal, country, year of publication, and sample size), patient characteristics (e.g., 
age, sex, body mass index, eligibility criteria, surgical type and surgical position), study design and quality (e.g., random sequence generation, allocation concealment and blinding methods), detail information of interventions and controls [e.g., conventional ventilation mode (PCV-VG, VCV or PCV), ventilation mode (One lung ventilation, two lung ventilation), intra-abdominal pressure during pneumoperitoneum, target value of tidal volume, inspiratory to expiratory ratio (I/E), respiratory rate (RR), $\mathrm{FiO}_{2}$, positive endexpiratory pressure (PEEP), target value of $\mathrm{PETCO}_{2}$ ], outcome measures, conflicts of interest, and any other relevant information. Third reviewer (GC) will double-check the data to ensure consistency.

If necessary, we will extract numerical data from graphs using Adobe Photoshop as described by Sevda Gheibi [41]. If data are missing or incomplete in any study, we will contact with the authors to obtain the original data. In case it is impossible to obtain the data, to ensure the statistical power and avoid bias, the last observation carried forward imputation method will be used to assume a missing value and then an intent-to-treat analysis and sensitivity analysis will be performed to assess whether that the results are consistent.

For continuous outcome data, the mean differences (MDs) or the standardised mean difference (SMDs) with $95 \%$ confidence intervals (Cls) will be used for analysis. For dichotomous data, the RR with $95 \% \mathrm{Cls}$ will be used for analysis.

\section{Assessment of risk of bias}

The risk of bias in the included studies will be determined by two reviewers (JW and LZ) independently using the Cochrane Collaboration tool [42]. The Cochrane Collaboration's tool covers six aspects, random sequence generation, allocation concealment, blinding of participants and personnel, blinding of outcome assessment, incomplete outcome data, selective reporting and other bias. The risk will be divided into three levels (low risk, unclear and high risk) in accordance with the item in the checklist. If any disagreements, the risk assignment will be settled through arbitration of a third reviewer (GC).

\section{Quality of evidence}

The modified Jadad scale will be applied to assess the quality of literatures $[43,44]$. The overall quality of included studies will be conducted in eight Items: randomization (with score 0-2), allocation concealment (with score 0-2), blinding (with score 0-2), withdrawals and dropouts (with score 0-1) adverse effects (with score 0-1), and statistical analysis (with score 0-1) (Table 2). Scale scores for each study could range from 0 to 8 points, with higher scores indicating better quality. Studies with a score less than 4 are defined as low-quality, while 4-8 are considered as high-quality.

Table 2 The modified Jaded Scale 


\section{Items}

1. Was the study described as randomized?

$\begin{array}{ll}\text { Yes } & 1\end{array}$

$\begin{array}{ll}\text { No } & 0\end{array}$

2. Was the method of randomization appropriate?

$\begin{array}{ll}\text { Yes } & 1\end{array}$

No

$\begin{array}{ll}\text { Not described } & 0\end{array}$

3. Was the study described as blinded?

Yes (Double-blind)

$\begin{array}{ll}\text { Yes (Single-blind) } & 0.5\end{array}$

$\begin{array}{ll}\text { No } & 0\end{array}$

4. Was the method of blinding appropriate?

Yes

No

Not described

5. Was there a description of withdrawals and dropouts?

Yes

No

6. Was there a clear description of the inclusion and exclusion criteria?

Yes

No

7. Was the method used to assess adverse effects described?

Yes

No

8. Was the method of statistical analysis described?

Yes

No 
Heterogeneity analyses will be performed by Review Manager version 5.4 (Rev Man, Cochrane Collaboration, Oxford, UK). Statistical heterogeneity will be assessed by standard $\chi^{2}$ test $(a=0.1)$ and $\mathrm{I}^{2}$ test. if the $p \geq 0.1$, and if $I^{2} \leq 50 \%$, the fixed-effects model will be used. if the $p<0.1$ or the $I^{2}>50 \%$, the random-effects models will be applied. When the heterogeneity is statistically significant, we will conduct a subgroup analysis to investigate the possible sources of heterogeneity according to the patient characteristics and the classification of surgery. If the $\mathrm{I}^{2}>75 \%$, a meta-analysis will not be performed and a narrative, qualitative summary will be provided.

\section{Subgroup analysis}

If data are available, subgroup analysis will be performed to assess the heterogeneity according to the conventional mechanical ventilation mode and types of surgery. To explore significant heterogeneity, if a sufficient number of trials are available, we will conduct the following prespecified subgroup analyses separately (hypothesised direction of effect in parentheses): (a) Type of conventional mechanical ventilation mode (PCV-VG is more effective in surgery as compared with PCV or VCV mode). (b) Type of surgery (PCV-VG is more effective in thoracic surgery with one-lung ventilation or laparoscopic gynecologic surgery with carbon dioxide pneumoperitoneum as compared with conventional mechanical ventilation modes).

\section{Trial Sequential Analysis}

Trial Sequential Analysis (TSA) will be conducted to control the risks of type I errors and type II errors [4547]. The required information size (RIS: the number of participants needed in a meta-analysis to detect or reject a certain intervention effect) and the cumulative Z-curve's breach of relevant trial sequential to monitor boundaries will be calculated for all outcomes [48-49]. For continuous outcomes, we will use the observed SD, a mean difference of the observed SD/2, an alpha of $2.5 \%$ and a beta of $10 \%$ for outcomes in the TSA [50]. For dichotomous outcomes, we will use the proportion of participants with an outcome in the control group, a relative risk reduction of $20 \%$, and an alpha of $2.5 \%$ and a beta of $10 \%$ in the TSA [51]. TSA will be performed using the TSA program version 0.9.5.10 Beta (http://www.ctu.dk/tsa) [52].

\section{Sensitivity analysis}

If possible, sensitivity analysis will be used to evaluate how uncertain assumptions of data and usage affect the robustness of the combined results. We will exclude low quality studies, re-analyse the included studies, and assess whether there are significant differences between the combined effects. If necessary, we will remove the included studies one by one from the pooled analyses.

\section{Assessment of reporting biases}

The potential publication bias will be statistically analysed using funnel plots analysis and Egger's regression test when more than 10 studies are involved for an outcome [53]. In the presence of plot asymmetry for a given analysis (e.g., publication bias), trim-and-fill method will be used to determine the 
impact of removing smaller studies on the overall estimate and provide adjusted results [54]. Reporting biases will be performed by Stata/MP 16.0 (Stata Corp, College Station, TX, USA).

\section{Discussion}

Mechanical ventilation has the potential risk to cause PPCs, which are the main cause of overall perioperative morbidity and mortality in patients. Optimization of mechanical ventilation could reduce PPCs, reduce global morbidity and mortality, improve the quality of medical care and decrease hospital costs. PCV-VG is a new mode introduced recently in the field of anesthesiology. It combines the advantages of VCV and PCV, which might improve arterial oxygenation, reduce inflammatory factors and ALI. However, whether PCV-VG could reduce the postoperative pulmonary complications remains uncertain.

This review might be the most comprehensive review about PCV-VG for PPCs. We will summarize the current evidence and providing valuable information for trial design in the future. Our systematic review and meta-analysis will examine the effect of PCV-VG compared with conventional mechanical ventilation modes on PPCs. This will help clinical practitioners in decision-making when choosing optimal intraoperative mechanical ventilation mode and support the development of clinical practice guidelines in the future.

\section{Strengths And Limitations}

This systematic review protocol follows the preferred reporting items for systematic review and metaanalysis protocols guidelines conducting a rigorous risk of bias assessment. We will use the modified Jadad Scale to assess the quality of evidence. TSA will be used to assess the optimal sample size for the outcomes and control the risk of random errors. Publication bias will be assessed by Funnel plots and Egger's regression test. Subgroup analysis will be performed to assess the heterogeneity according to the conventional mechanical ventilation mode and types of surgery.

There are also limitations to our analysis. First, studies with different mechanical ventilation time, different value of preset tidal volume and different value of preset pressure will be included, resulting in potential heterogeneity. Second, the sample size in each study may be small. Third, number of studies with eligible data for subgroup analyses may be limited. Fourth, another limitation may be the current lack of high-level evidence, such as well-designed randomized controlled trials with double-blind.

\section{Abbreviations}

PPCs: Postoperative pulmonary complications; PCV-VG: Pressure-controlled ventilation-volume guaranteed; VCV: Volume-controlled ventilation; PCV: Pressure-controlled ventilation; VALI: Ventilatorassociated lung injury; ALI: Acute lung injury; PIP: Peak inspiratory pressure; $\mathrm{CO}_{2}$ : Carbon dioxide; PRVC: Pressure regulated volume controlled; I/E: Inspiratory to expiratory ratio; RR: Respiratory rate; PEEP: 
positive end-expiratory pressure; $\mathrm{PaO}_{2}$ : arterial partial pressure of oxygen; $\mathrm{SaO}_{2}$ : arterial oxygen saturation; $\mathrm{PaCO}_{2}$ : arterial partial pressure of carbon dioxide; $\mathrm{PEtCO}_{2}$ : pressure of end-tidal carbon dioxide; $\mathrm{FiO}_{2}$ : fraction of inspiration $\mathrm{O}_{2} ; \mathrm{V}_{\mathrm{D}}$ : Dead space volume; $\mathrm{V}_{\mathrm{T}}$ : Tidal volume; $\mathrm{P}_{\text {peak }}$ : Peak inspiratory pressure; $P_{\text {mean }}$ : mean inspiratory pressure; $P_{\text {plateau }}$ : plateau inspiratory pressure; $C_{\text {dyn }}$ : pulmonary dynamic compliance; MAP: Mean arterial pressure; HR: Heart rate; CVP: Central venous pressure; PRISMAP: Preferred Reporting Items for Systematic Reviews and Meta-Analysis Protocol; RCTs: Randomized controlled trials; Cls: Confidence intervals; MDs: Mean differences; SMD: Standardized MDs; RR: Risk ratio; EPCO: European Perioperative Clinical Outcome; TSA: Trial Sequential Analysis; RIS: Required information size.

\section{Declarations}

\section{Ethics approval and consent to participate}

Ethical approval is not applicable as this is a systematic review protocol; we will not involve the human population directly.

\section{Consent for publication}

Not applicable as the manuscript does not contain data from any person.

\section{Availability of data and materials}

The data acquisition for this systematic review has not yet started. We plan to conduct the search in midMay. Subsequently, dataset generated through this systematic review can be requested from the corresponding author.

\section{Competing interests}

The authors declare no competing interests.

\section{Funding}

This study was supported by the Sichuan Science and Technology Program (Grant No.2019YJ0058). JQZ is supported by Sichuan Science and Technology Program (Grant No. 2019YJ0058). The funder has no role in

developing this protocol.

\section{Authors' contributions}

JQZ and LD conceived the idea for this systematic review. All authors (JQZ, LD, JW, LZ, XQD, GC) developed the methodology for the systematic review. The manuscript was drafted by JQZ, LD, and revised by all authors. XQD and GC will screen potential studies, perform duplicate independent data 
abstraction. JQZ and LZ will undertake risk of bias assessment and assess the evidence quality. JQZ and LD will conduct the data synthesis. All authors contributed to the research and agreed to be responsible for all aspects of the work. GC is the guarantor of the review.

\section{Acknowledgements}

Not applicable.

\section{Authors' information}

${ }^{1}$ Department of Anesthesiology, Sichuan University West China Hospital, No. 37th, Guoxue Alley, Wuhou District, Chengdu 610041, Sichuan, China. ${ }^{2}$ Department of Anesthesiology, Sichuan Cancer Hospital \& Institute, Sichuan Cancer Center, School of Medicine, University of Electronic Science and Technology of China, No.55th, People's South Road, Chengdu 610041, Sichuan, China.

\section{References}

1. Meara JG, Leather AJM, Hagander L, Alkire BC, Alonso N, Ameh EA, et al. Global Surgery 2030: evidence and solutions for achieving health, welfare, and economic development. Lancet 2015;386 (9993): 569-624.

2. Fernandez-Bustamante A, Frendl G, Sprung J, Kor DJ, Subramaniam B, Martinez Ruiz R, et al. Postoperative Pulmonary Complications, Early Mortality, and Hospital Stay Following Noncardiothoracic Surgery: A Multicenter Study by the Perioperative Research Network Investigators. JAMA Surg. 2017; 152 (2):157-166.

3. Ball L, Hemmes SNT, Serpa Neto A, Bluth T, Canet J, Hiesmayr M, et al. Intraoperative ventilation settings and their associations with postoperative pulmonary complications in obese patients. $\mathrm{Br} J$ Anaesth 2018; 121(4): 899-908.

4. Canet J, Gallart L, Gomar C, Paluzie G, Vallès J, Castillo J, et al. Prediction of postoperative pulmonary complications in a population-based surgical cohort. Anesthesiology 2010; 113 (6):13381350.

5. VEGAS Investigators LAS. Epidemiology, practice of ventilation and outcome for patients at increased risk of postoperative pulmonary complications: LAS VEGAS dan observational study in 29 countries. Eur J Anaesthesiol 2017; 34 (8): 492-507.

6. Khuri SF, Henderson WG, DePalma RG, Mosca C, Healey NA, Kumbhani DJ, et al. Determinants of long-term survival after major surgery and the adverse effect of postoperative complications. Ann Surg. 2005; 242 (3):326-41, discussion 341-3.

7. Shander A, Fleisher LA, Barie PS, Bigatello LM, Sladen RN, Watson CB. Clinical and economic burden of postoperative pulmonary complications: patient safety summit on definition, risk-reducing interventions, and preventive strategies. Crit Care Med. 2011; 39(9):2163-72. 
8. Guldner A, Kiss T, Serpa Neto A, Hemmes SN, Canet J, Spieth PM, et al. Intraoperative protective mechanical ventilation for prevention of postoperative pulmonary complications: a comprehensive review of the role of tidal volume, positive end-expiratory pressure, and lung recruitment maneuvers. Anesthesiology. 2015;123(3): 692-713.

9. Miskovic A, Lumb AB. Postoperative pulmonary complications. Br J Anaesth. 2017;118(3):317-334.

10. Young CC, Harris EM, Vacchiano C, Bodnar S, Bukowy B, Elliott RRD, et al. Lung-protective ventilation for the surgical patient: international expert panel-based consensus recommendations. $\mathrm{Br} \mathrm{J}$ Anaesth. 2019;123 (6): 898-913.

11. Ball L, Dameri M, Pelosi P. Modes of mechanical ventilation for the operating room. Best Pract Res Clin Anaesthesiol.2015; 29(3): 285-299.

12. Pu J, Liu Z, Yang L, Wang Y, Jiang J. Applications of pressure control ventilation volume guaranteed during one-lung ventilation in thoracic surgery. Int J Clin Exp Med. 2014;7(4): 1094-8

13. Şenay H, Sıvacı R, Kokulu S, Koca B, Bakı ED, Ela Y. The effect of pressure-controlled ventilation and volume-controlled ventilation in prone position on pulmonary mechanics and inflammatory markers. Inflammation. 2016; 39 (4):1469-74.

14. Maeda Y, Fujino Y, UchiyamaA, Matsuura N, Mashimo T, Nishimura M. Effects of peak inspiratory flow on development of ventilator-induced lung injury in rabbits. Anesthesiology. 2004; 101(3):722-8.

15. Nichols D, Haranath S. Pressure Control Ventilation. Crit Care Clin. 2007; 23(2): 183-99.

16. Prella M, Feihl F, Domenighetti G. Effects of short-term pressure-controlled ventilation on gas exchange, airway pressures, and gas distribution in patients with acute lung injury/ARDS: Comparison with volume-controlled ventilation. Chest. 2002; 122(4):1382-8.

17. Park JH, Park IK, Choi SH, Eum D, Kim MS. Volume-Controlled Versus Dual-Controlled Ventilation during Robot-Assisted Laparoscopic Prostatectomy with Steep Trendelenburg Position: A Randomized-Controlled Trial. J Clin Med. 2019; 8(12):2032.

18. Cinnella G, Grasso S, Spadaro S, Rauseo M, Mirabella L, Salatto P, et al. Effects of recruitment maneuver and positive end-expiratory pressure on respiratory mechanics and transpulmonary pressure during laparoscopic surgery. Anesthesiology. 2013; 118 (1): 114-22.

19. Kothari A, Baskaran D. Pressure-controlled volume guaranteed mode improves respiratory dynamics during laparoscopic cholecystectomy: a comparison with conventional modes. Anesth Essasys Res. 2018;12(1): 206-12.

20. Keszler M. Volume-targeted ventilation. Early Hum Dev. 2006; 82(12): 811-8.

21. Dion JM, McKee C, Tobias JD, Sohner P, Herz D, Teich S, et al. Ventilation during laparoscopicassisted bariatric surgery: volume-controlled, pressure-controlled or volume-guaranteed pressureregulated modes. Int J Clin Exp Med. 2014; 7(8):2242-7.

22. Keszler M, Abubakar K. Volume guarantee: Stability of tidal volume and incidence of hypocarbia. Pediatr Pulmonol. 2004; 38(3):240-5. 
23. Kallet RH, Campbell AR, Dicker RA, Katz JA, Mackersie RC. Work of breathing during lung-protective ventilation in patients with acute lung injury and acute respiratory distress syndrome: a comparison between volume and pressure-regulated breathing modes. Respir Care. 2005; 550(12):1623-31.

24. Chang S, Shi J, Fu C, Wu X, Li S. A comparison of synchronized intermittent mandatory ventilation and pressure-regulated volume control ventilation in elderly patients with acute exacerbations of COPD and respiratory failure. Int J Chron Obstruct Pulmon Dis. 2016; 11:1023-9.

25. D'Angio CT, Chess PR, Kovacs SJ, Sinkin RA, Phelps DL, Kendig JW, et al. Pressure-regulated volume control ventilation vs synchronized intermittent mandatory ventilation for very low-birthweight infants: a randomized controlled trial. Arch Pediatr Adolesc Med. 2005; 159 (9):868-75.

26. Gad M, Gaballa K, Abdallah A, Abdelkhalek M, Zayed A, Nabil H. Pressure-controlled ventilation with volume guarantee compared to volume-controlled ventilation with equal ratio in obese patients undergoing laparoscopic hysterectomy. Anesth Essays Res 2019; 13(2):347-53.

27. Lin F, Pan L, Huang B, Ruan L, Liang R, Qian W, et al. Pressure-controlled versus volume-controlled ventilation during one-lung ventilation in elderly patients with poor pulmonary function. Ann Thorac Med. 2014; 9 (4): 203-8.

28. Tugrul M, Camci E, Karadeniz H, Sentürk M, Pembeci K, Akpir K. Comparison of volume controlled with pressure controlled ventilation during one-lung anaesthesia. $\mathrm{Br} J$ Anaesth.1997; 79(3): 306-10.

29. Senturk NM, Dilek A, Camci E, Sentürk E, Orhan M, Tuğrul M, et al. Effects of positive end-expiratory pressure on ventilatory and oxygenation parameters during pressure-controlled one-lung ventilation. J Cardiothorac Vasc Anesth. 2005; 19(1): 71-5.

30. Heimberg C, Winterhalter M, Struber M, Piepenbrock S, Bund M. Pressure-controlled versus volumecontrolled one-lung ventilation for MIDCAB. Thorac Cardiovasc Surg. 2006; 54(8): 516-20.

31. Senturk M. New concepts of the management of one-lung ventilation. Curr Opin Anaesthesiol. 2006; 19(1): 1-4.

32. Pardos PC, Garutti I, Pineiro P, Olmedilla L, de la Gala F. Effects of ventilatory mode during onelung ventilation on intraoperative and postoperative arterial oxygenation in thoracic surgery. $\mathrm{J}$ Cardiothorac Vasc Anesth. 2009; 23(6): 770-4.

33. Assad OM, El Sayed AA, Khalil MA. Comparison of volume-controlled ventilation and pressurecontrolled ventilation volume guaranteed during laparoscopic surgery in Trendelenburg position. $J$ Clin Anesth.2016; 34:55-61.

34. Choi EM, Na S, Choi SH, An J, Rha KH, Oh YJ. Comparison of volume-controlled and pressurecontrolled ventilation in steep Trendelenburg position for robot-assisted laparoscopic radical prostatectomy. J Clin Anesth. 2011; 23(3):183-8.

35. Lian M, Zhao X, Wang H, Chen L, Li S. Respiratory dynamics and dead space to tidal volume ratio of volume-controlled versus pressure-controlled ventilation during prolonged gynecological laparoscopic surgery. Surg Endosc. 2017;31(9):3605-3613.

36. Liao CC, Kau YC, Ting PC, Tsai SC, Wang CJ. The effects of volume-controlled and pressurecontrolled ventilation on lung mechanics, oxidative stress, and recovery in gynecologic laparoscopic 
surgery. J Minim Invasive Gynecol. 2016; 23 (3):410-7.

37. Liberati A, Altman DG, Tetzlaff J, Mulrow C, Gøtzsche PC, loannidis JP, et al. The PRISMA statement for reporting systematic reviews and meta-analyses of studies that evaluate health care interventions: explanation and elaboration. PLoS Med. 2009; 6(7): e1000100.

38. Shamseer L, Moher D, Clarke M, Ghersi D, Liberati A, Petticrew M, et al. Preferred reporting items for systematic review and meta-analysis protocols (PRISMA-P) 2015: elaboration and explanation. BMJ. 2015; 350:

39. Jammer I, Wickboldt N, Sander M, Smith A, Schultz MJ, Pelosi P, et al. Standards for definitions and use of outcome measures for clinical effectiveness research in perioperative medicine: European Perioperative Clinical Outcome (EPCO) definitions: a statement from the ESA-ESICM joint taskforce on perioperative outcome measures. Eur J Anaesthesiol. 2015;32(2):88-105.

40. Abbott TEF, Fowler AJ, Pelosi P, Gama de Abreu M, Møller AM, et al. A systematic review and consensus definitions for standardized end-points in perioperative medicine: pulmonary complications. Br J Anaesth. 2018; 120(5):1066-79.

41. Gheibi S, Mahmoodzadeh A, Kashfi K, Jeddi S, Ghasemi A. Data Extraction from Graphs Using Adobe Photoshop: Applications for Meta-Analyses. Int J Endocrinol Metab. 2019;17(4): e95216.

42. Higgins JPT, Altman DG, Gøtzsche PC, Jüni P, Moher D, Oxman AD, et al. The Cochrane collaboration's tool for assessing risk of bias in randomised trials. BMJ. 2011; 343: d5928.

43. Jadad AR, Moore RA, Carroll D, Jenkinson C, Reynolds DJ, Gavaghan DJ, et al. Assessing the quality of reports of randomized clinical trials: is blinding necessary? Control Clin Trials.1996; 17(1):1-12.

44. Oremus M, Wolfson C, Perrault A, Demers L, Momoli F, Moride Y. Interrater reliability of the modified Jadad quality scale for systematic reviews of Alzheimer's disease drug trials. Dement Geriatr Cogn Disord. 2001;12(3): 232-6.

45. Wetterslev J, Thorlund K, Brok J, Gluud C. Estimating required information size by quantifying diversity in random-effects model meta-analyses. BMC Med Res Methodol.2009; 9:86.

46. Wetterslev J, Jakobsen JC, Gluud C. Trial sequential analysis in systematic reviews with metaanalysis. BMC Med Res Methodol. 2017; 17(1):39.

47. Wetterslev J, Thorlund K, Brok J, Gluud C. Trial sequential analysis may establish when firm evidence is reached in cumulative meta-analysis. J Clin Epidemiol. 2008; 61(1): 64-75.

48. Imberger G, Thorlund K, Gluud C, Wetterslev J. False-Positive findings in Cochrane meta-analyses with and without application of trial sequential analysis: an empirical review. BMJ Open.2016;6 (8): e011890.

49. Brok J, Thorlund K, Gluud C, Wetterslev J. Trial sequential analysis reveals insufficient information size and potentially false positive results in many meta-analyses. J Clin Epidemiol. 2008; 61(8):7639.

50. Norman GR, Sloan JA, Wyrwich KW. Interpretation of changes in health-related quality of life: the remarkable universality of half a standard deviation. Med Care. 2003; 41(5):582-92. 
51. Castellini G, Nielsen EE, Gluud C. Comment on: "Cell therapy for heart disease: Trial sequential analyses of two cochrane reviews". Clin Pharmacol Ther. 2017; 102(1):21-4.

52. Thorlund K, Engstrøm J, Wetterslev J, Brok J, Imberger G, Gluud C. User manual for trial sequential analysis (TSA) Copenhagen trial unit, centre for clinical intervention research, Denmark; 2011. Available from:https://ctu.dk/wp-content/uploads/2021/03/2017-10-10-TSA-Manual-ENG_ER.pdf. Accessed 2021.

53. Egger M, Davey Smith G, Schneider M, Minder C. Bias in meta-analysis detected by a simple, graphical test. BMJ. 1997; 315 (7109): 629-634.

54. Duval S, Tweedie R. Trim and fill: a simple funnel-plot-based method of testing and adjusting for publication bias in meta-analysis. Biometrics. 2000;56(2):455-463.

\section{Figures}



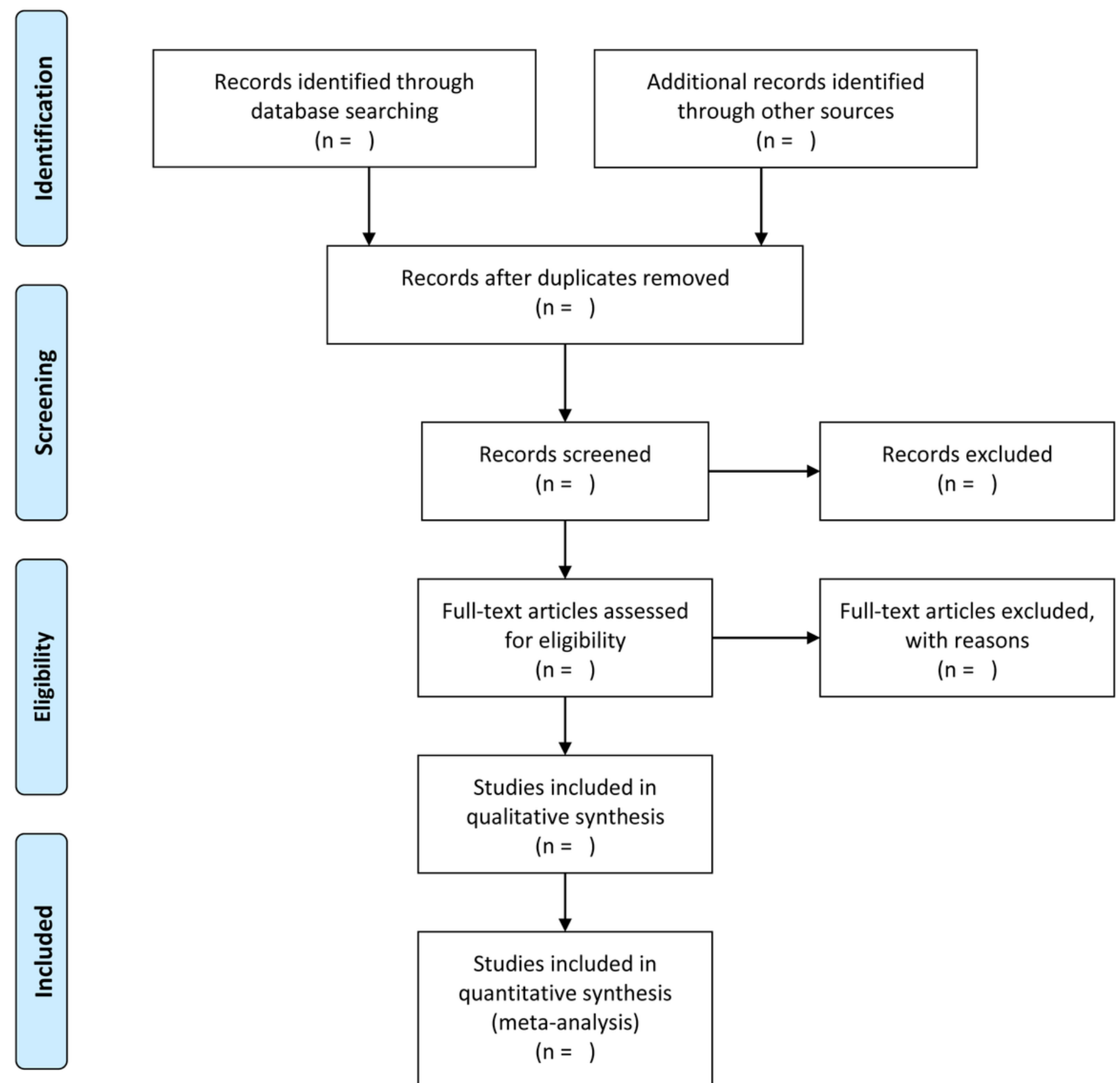

Studies included in qualitative synthesis

$$
(n=)
$$

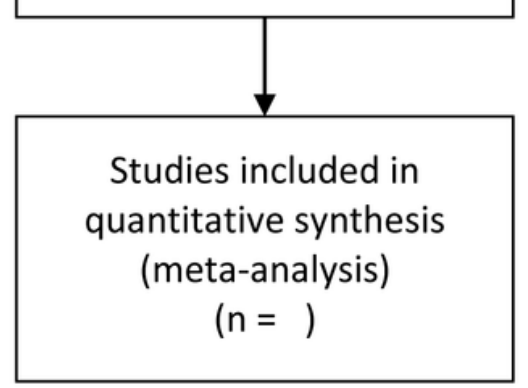

Figure 1

PRISMA flow diagram of study and exclusion. PRISMA, Preferred Reporting Items for Systematic Reviews and Meta-analysis.

\section{Supplementary Files}

This is a list of supplementary files associated with this preprint. Click to download. 
- Additionalfile1PRISMAP2015Checklist.docx

- Additionalfile2SearchStrategy.docx

- Additionalfile3Draftdataextractionform.docx 